\section{The level of peripheral regulatory $T$ cells is linked to changes in gut commensal microflora in patients with systemic lupus erythematosus}

Systemic lupus erythematosus (SLE) is a multisystem autoimmune disorder mainly mediated by lymphocytes and autoantibodies, which have a pervasive negative impact on the majority of organs. ${ }^{12}$ It deserves more attention to further explore the pathogenesis because of the unclear complex pathogenesis and the limited clinical efficacy of SLE treatment.

Recently, a cross-sectional discovery cohort in the USA conducted by Azzouz et al, published in Annals of Rheumatic Diseases, suggested that specific gut commensal strains, especially Ruminococcus gnavus, may contribute to the disease activity and autoantibody production in SLE patients, ${ }^{3}$ which put forward a novel concept for the immune pathogenesis of SLE. Their study was well performed and analysed the abundance of intestinal flora and sera profiled for antibacterial and autoantibody responses between SLE patients with the different disease activity index scores and matched healthy controls. However, the faecal microbiota is lacking a precise link with lymphocytes in peripheral blood of the patients, which are the important participants of immune mechanism of SLE.

In this study, we studied the correlation between the changes in faecal microbial diversity and the absolute numbers of peripheral lymphocyte subgroups and CD4 + T subsets in SLE patients, especially regulatory $\mathrm{T}$ cells (Tregs) that mediate immune tolerance and maintain immunological homeostasis. ${ }^{4}$ The blood and stool samples were collected from 92 patients with SLE and 217 matched healthy adults. The $16 \mathrm{~S}$ rRNA in the stool specimens were sequenced using the Roche/45 high-throughput sequencing platform. The absolute numbers of circulating lymphocytes and CD4+ T subgroups of these individuals were detected by flow cytometry combined with standard absolute counting beads. ${ }^{5}$

Patients with SLE, regardless male or female, had different taxonomic diversity and abundance of specific strains of a gut commensal at the level of the phylum, family and genus $(\mathrm{p}<0.05)$ from healthy controls. They had higher levels of Proteobacteria, Bacteroidetes and Actinobacteria and a lower level of Firmicutes as compared with those of healthy controls at the phylum level $(\mathrm{p}<0.05)$. In addition, at the family or genus level, the proportion or abundance of gut bacteria in SLE patients, including Bacteroidaceae, Veillonellaceae, Klebsiella, Streptococcaceae and Erysipelotrichaceae, differed from those in healthy individuals with statistically significant difference $(p<0.05)($ table 1$)$. It was noteworthy that patients with SLE had significantly lower proportion of Ruminococcaceae at family level than healthy controls $(\mathrm{p}<0.001)$. Interestingly, the percentage of Ruminococcus at genus level was higher in SLE patients $(\mathrm{p}<0.05)$, which was confirmed to involved in the incidence of lupus in Azzouz et al's study, suggesting that we should study the related bacterial flora and its mechanism of SLE patients at multiple levels.

We found that the proportion of Ruminococcus was correlated with the absolute counts of lymphocytes, suggesting that the changed of intestinal flora was involved in the imbalance of proinflammation and anti-inflammation T cells in SLE. Particularly, the proportion of Ruminococcus was significantly correlated with the absolute counts of Tregs (its dysfunction was one of the crucial immune mechanisms in the onset of lupus) ${ }^{6}$ and the ratio of Th1/Th2 and Th17/Treg (figure 1D-F), but not with the numbers of Th1, Th2 and Th17 cells (figure 1A-C), which may be one of the reasons for the changes in intestinal
Table 1 Shifts in taxonomic abundance between SLE and healthy controls (HC) $($ mean \pm SD)

\begin{tabular}{lccc}
\hline Taxonomy & SLE (\%) & HC (\%) & P value \\
\hline Phylum & & & \\
\hline Proteobacteria & $13.26 \pm 21.77$ & $2.65 \pm 3.56$ & $<0.001$ \\
\hline Firmicutes & $40.14 \pm 25.58$ & $59.20 \pm 19.19$ & $<0.001$ \\
\hline Bacteroidetes & $42.69 \pm 25.26$ & $35.75 \pm 18.58$ & 0.018 \\
\hline Actinobacteria & $2.25 \pm 3.49$ & $1.26 \pm 3.28$ & 0.002 \\
\hline Family & & & \\
\hline Lachnospiraceae & $16.35 \pm 13.09$ & $25.09 \pm 12.89$ & $<0.001$ \\
\hline Ruminococcaceae & $12.72 \pm 12.68$ & $23.50 \pm 13.57$ & $<0.001$ \\
\hline Bacteroidaceae & $35.12 \pm 25.56$ & $26.67 \pm 18.03$ & 0.021 \\
\hline Veillonellaceae & $2.63 \pm 5.42$ & $5.68 \pm 9.30$ & $<0.001$ \\
\hline Streptococcaceae & $1.26 \pm 5.56$ & $0.24 \pm 1.49$ & $<0.001$ \\
\hline Genus & & & \\
\hline Ruminococcus & $2.63 \pm 6.59$ & $1.41 \pm 2.04$ & 0.016 \\
\hline Haemophilus & $0.15 \pm 0.53$ & $0.17 \pm 0.59$ & 0.035 \\
\hline Faecalibacterium & $7.58 \pm 9.80$ & $19.99 \pm 13.87$ & $<0.001$ \\
\hline Bacteroides & $38.75 \pm 26.96$ & $30.61 \pm 19.8$ & 0.016 \\
\hline Clostridium IV & $0.17 \pm 0.40$ & $0.27 \pm 0.51$ & $<0.001$ \\
\hline Klebsiella & $3.04 \pm 9.39$ & $0.30 \pm 1.54$ & 0.001 \\
\hline Erysipelotrichaceae & $0.06 \pm 0.32$ & $0.02 \pm 0.13$ & $<0.001$ \\
\hline StE systemic lupus & & \\
\hline
\end{tabular}

SLE, systemic lupus erythematosus.

microbial population are involved in SLE patients. However, there was no obvious correlation between the abundance of Ruminococcus and the absolute numbers of total T, B, natural killer (NK), CD4+ T and CD8 + T cells ( $\mathrm{p}>0.05)$.

In conclusion, the changes in taxonomic diversity and the abundance of partial specific flora, such as Ruminococcus genus, may regulate the absolute number of Tregs in peripheral blood to participate in the pathogenesis of SLE. This study on the correlation between the intestinal flora abundance and levels

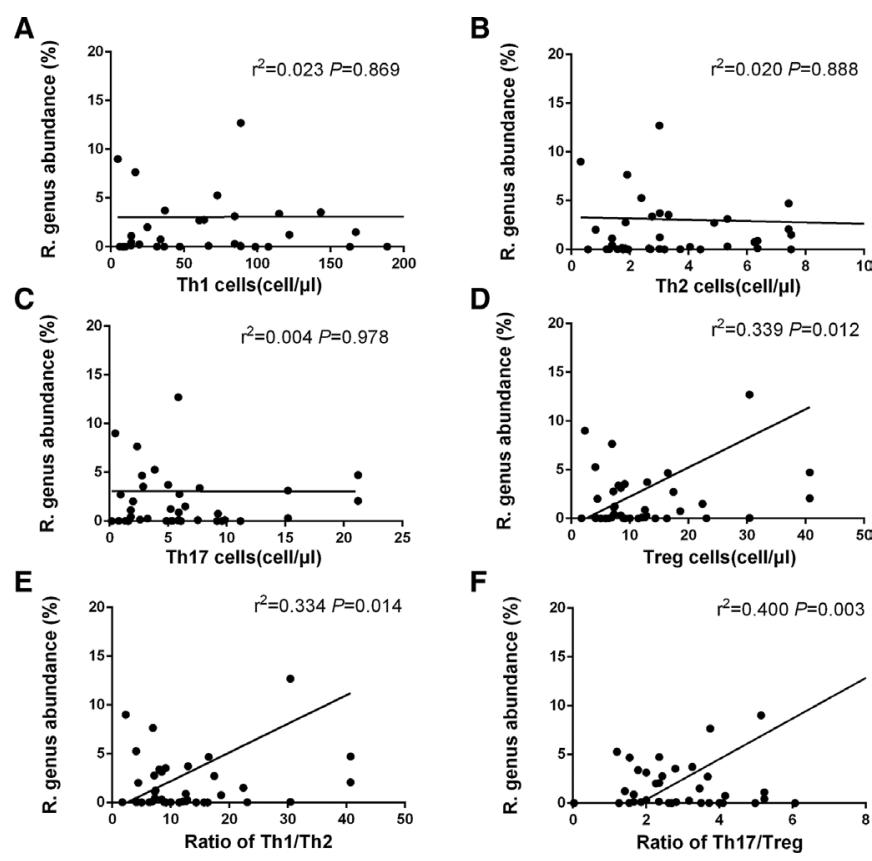

Figure 1 Correlation of absolute numbers of CD4+ T subsets and the ratio of Th1/Th2 and Th17/Treg with the propotion of Ruminococcus at genus level in SLE patients by Spearman coefficient. All $p$ values reported herein are two-tailed. $\mathrm{P}<0.05$ was taken as statistical significance. SLE, systemic lupus erythematosus. 
of lymphocyte subsets reconfirmed the involvement of gut commensal microflora in the pathogenesis of SLE.

Sheng-Xiao Zhang $\odot{ }^{1,2}$ Jia Wang, ${ }^{1,2}$ Jun-Wei Chen, ${ }^{2}$ Ming-Xing Zhang, ${ }^{2}$ Yi-Fan Zhang, ${ }^{3}$ Fang-Yuan Hu, ${ }^{2}$ Zhi-Qin Lv, ${ }^{2}$ Chong Gao, ${ }^{4}$ Ya-Feng Li, ${ }^{5}$ Xiao-Feng $\mathrm{Li}^{1,2}$

'Department of Rheumatology, Shanxi Li Xiaofeng Medical Groups, Taiyuan, China 'Department of Rheumatology, The Second Hospital of Shanxi Medical University, Taiyuan, China

${ }^{3}$ Department of Clinical Medicine, Shanxi Medical University, Taiyuan, China

${ }^{4}$ Department of Pathology, Brigham and Women's Hospital, Harvard Medical School, Boston, Massachusetts, USA

${ }^{5}$ Department of Nephrology, Precision Medicine Center, The Shanxi Provincial People's Hospital, Shanxi Medical University, Taiyuan, China

Correspondence to Professor Xiao-Feng Li; Ixf_9859@sxmu.edu.cnDr Ya-Feng Li; Dr.yafengli@gmail.com

Contributors Study design and manuscript writing: S-XZ and JW. Data extraction, quality assessment, analysis and interpretation of data: S-XZ, JW and JC. All authors were involved in drafting the article or revising it critically for important intellectual content, and all authors approved the final version to be published. Y-FL and XL had full access to all of the data in the study and take responsibility for the integrity of the data and the accuracy of the data analysis.

Funding This work was supported by a grant from the National Natural Science Foundation of China (81871295).

Competing interests None declared.

Patient consent for publication Not required.

Provenance and peer review Not commissioned; internally peer reviewed.

(c) Author(s) (or their employer(s)) 2019. No commercial re-use. See rights and permissions. Published by BMJ.

S-XZ and JW contributed equally.

\section{Check for updates}

To cite Zhang S-X, Wang J, Chen J-W, et al. Ann Rheum Dis Epub ahead of print: [please include Day Month Year]. doi:10.1136/annrheumdis-2019-216504

Received 20 October 2019

Accepted 22 October 2019

\section{Linked}

http://dx.doi.org/10.1136/annrheumdis-2019-216523

Ann Rheum Dis 2019:0:1-2. doi:10.1136/annrheumdis-2019-216504

\section{ORCID iD}

Sheng-Xiao Zhang http://orcid.org/0000-0003-1341-7588

\section{REFERENCES}

1 Vasquez-Canizares N, Wahezi D, Putterman C. Diagnostic and prognostic tests in systemic lupus erythematosus. Best Pract Res Clin Rheumatol 2017;31:351-63.

2 Mohan C, Putterman C. Genetics and pathogenesis of systemic lupus erythematosus and lupus nephritis. Nat Rev Nephrol 2015:11:329-41.

3 Azzouz D, Omarbekova A, Heguy A, et al. Lupus nephritis is linked to diseaseactivity associated expansions and immunity to a gut commensal. Ann Rheum Dis 2019;78:947-56.

4 He J, Zhang $X$, Wei Y, et al. Low-dose interleukin-2 treatment selectively modulates CD4(+) T cell subsets in patients with systemic lupus erythematosus. Nat Med 2016;22:991-3.

5 Miao M, Hao Z, Guo Y, et al. Short-Term and low-dose IL-2 therapy restores the Th17/ Treg balance in the peripheral blood of patients with primary Sjögren's syndrome. Ann Rheum Dis 2018;77:1838-40.

6 Zhang S-X, Ma X-W, Li Y-F, et al. The proportion of regulatory T cells in patients with systemic lupus erythematosus: a meta-analysis. J Immunol Res 2018;2018:1-11. 